\title{
Topological phase diagram of a three-terminal Josephson junction: From the conventional to the Majorana regime
}

\author{
Lucila Peralta Gavensky, Gonzalo Usaj, and C. A. Balseiro \\ Centro Atómico Bariloche and Instituto Balseiro, Comisión Nacional de Energía Atómica (CNEA)- \\ Universidad Nacional de Cuyo (UNCUYO), 8400 Bariloche, Argentina \\ and Instituto de Nanociencia y Nanotecnología (INN-Bariloche), Consejo Nacional de Investigaciones \\ Científicas y Técnicas (CONICET), Argentina
}

(Received 22 April 2019; published 16 July 2019)

\begin{abstract}
We study the evolution of averaged transconductances in three-terminal Josephson junctions when the superconducting leads are led throughout a topological phase transition from an $s$-wave to a $p$-wave (Majorana) phase by an in-plane magnetic field $B_{x}$. We provide a complete description of this transition as a function of $B_{x}$ and a magnetic flux $\Phi$ threading the junction. For that we use a spinful model within a formalism that allows us to treat on an equal footing the contribution to the transconductance from both the Andreev subgap levels and the continuum spectrum. We unveil a fractionalization in the quantization of the transconductance due to the presence of Majorana quasiparticles, reflecting the effective pumping of half a Cooper pair charge in the $p$-wave regime.
\end{abstract}

DOI: 10.1103/PhysRevB.100.014514

\section{INTRODUCTION}

The quest for finding novel topological properties in condensed-matter systems has drawn increasing attention both in theoretical and experimental research. Much of the progress in the field has relied on the idea of the emergence of topological features by engineering heterostructures of conventional materials [1-4], driving them out of equilibrium [5-7] or by the direct design of topological phases with cold-atom systems [8-11]. In this way, it is now possible to artificially mimic topological matter without the need of working with exotic materials per se.

Following this line of thought, multiterminal Josephson junctions of at least four conventional superconductors coupled through a normal scattering region have been put forward as an exciting platform to engineer Weyl-topological phases [12]. The existence of Weyl singularities in the spectrum of multiply connected networks can be traced back to the seminal work by Avron et al. [13], who envisioned the possibility of nontrivial quantization of averaged transconductances (ATCs) in these devices. Different Josephson junction (JJ) circuits were also suggested as practical realizations to experimentally probe topologically protected adiabatic transport in these artificial setups [14].

More recently, it was shown that three-terminal structures can also develop topological properties when piercing the central scattering region with an external magnetic flux $[15,16]$ or when driven with an external microwave field [17]. There have also been proposals to measure the Fubini-Study geometric tensor [18] and the Berry curvature [17,18] of a multiterminal $\mathrm{JJ}$ using microwaves. Remarkably, all this theoretical activity has been fueled by several stimulating experiments on multiterminal superconducting devices [19-22].

In a multiterminal JJ, ATCs can be experimentally probed by voltage biasing one of the superconducting reservoirs as a function of a control parameter and measuring the phase-averaged current in a different lead. For conventional $s$-wave pairing, this would lead to a transconductance quantization in units of $4 e^{2} / h$ [23]. This outcome can be simply thought of as a nontrivial adiabatic charge pumping between leads, say $v$ and $v^{\prime}$, a product of the existence of Weyl sources in the spectrum $[12,13]$. For vanishing voltage $V$, the ATC results in $G_{v v^{\prime}}=Q_{P} /(V \Delta T)=n_{v v^{\prime}} 2 e q^{\star} / h$, where the pumping period $\Delta T$ is merely the Josephson period $h / 2 \mathrm{eV}$ and the phase-averaged pumped charge $Q_{P}$ over this period is an integer multiple $\left(n_{\nu v^{\prime}} \in \mathbb{Z}\right)$ of the effective charge of the Cooper pair $q^{\star}=2 e$.

A natural follow-up question is whether multiterminal JJs with $p$-wave pairing that host Majorana fermions may also develop a quantized ATC. Interestingly enough, this would provide a robust measurement of the effective tunneling charge in the topological superconductors, which is expected to be $q^{\star}=$ $e$ as in the case of the $4 \pi$-periodic fractional Josephson effect [24-27]. A partial answer using the Kitaev spinless model was recently found in Ref. [28] for the case of a four-terminal device (see also [29]). Yet, a complete description of the transition from the $s$-wave to the $p$-wave regime within a spinful approach in the entire parameter space is still missing. This is a necessary condition to obtain a unified topological phase diagram that could be experimentally tested. Even more, the calculation of the topological invariant associated with the ATC has so far been mainly based on the Chern numbers of the Andreev bound states (ABS) [12,16,28-30] despite the fact that in many regions of the parameter space they cannot be disentangled from the continuum spectrum above the superconducting gap (which might also contribute to the ATC [15]).

In this work, we present the complete topological phase diagram of a three-terminal JJ obtained by a direct calculation of the adiabatic corrections to the Josephson current. We use the general approach developed in Ref. [17], which includes the contributions from both the $\mathrm{ABS}$ and the continuum 


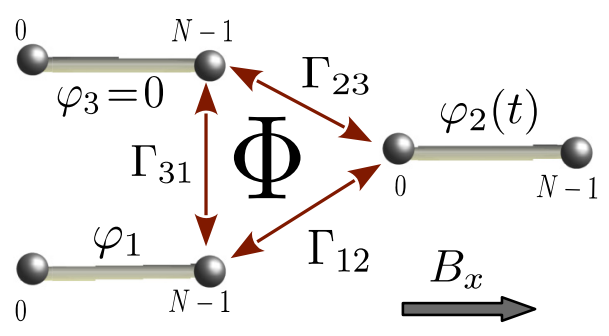

FIG. 1. Scheme of a Josephson trijunction threaded by an external magnetic flux $\Phi$. The superconducting leads (with phases $\varphi_{\nu}$ ) have Rashba spin-orbit coupling and can be driven into a topological (Majorana) regime by an in-plane magnetic field $B_{x}$.

spectrum. To describe the $s$-wave to $p$-wave transition, we consider a junction formed by spinful one-dimensional (1D) wires with Rashba spin-orbit coupling, proximity-induced superconductivity, and an in-plane magnetic field $B_{x}$ (see Fig. 1). This model, introduced in Refs. [24,25], is known to have a topological phase transition that allows Majorana fermions to develop at the end of the wires for $B_{x}>B_{c}$, with $B_{c}$ a critical field. An external magnetic flux $\Phi$ threading the central region of the device allows a fine tuning of the topological properties. For $B_{x}<B_{c}$ ( $s$-wave phase), we recover the usual quantization $\left(4 e^{2} / h\right)$ whenever the system is gapped, while for $B_{x}>B_{c}$ ( $p$-wave phase) we find a quantization in $2 e^{2} / h$ that we identify with the development of effective spinlesslike degrees of freedom, which make the measurement of a fractionalized pumped charge $\left(q^{\star}=e\right)$ possible. All these results are summarized in Fig. 2.

\section{MODEL HAMILTONIAN}

The Hamiltonian of the Josephson trijunction is given by $\hat{H}=\sum_{v=1}^{3} \hat{H}_{v}+\hat{H}_{\mathcal{T}}$, where $\hat{H}_{v}$ is the Hamiltonian of the lead $v$, and $\hat{H}_{\mathcal{T}}$ describes the Josephson tunneling. We use a spatially discretized version of the continuum model $[24,25]$ with $N \rightarrow \infty$ lattice sites to describe the leads:

$$
\hat{H}_{\nu}=\frac{1}{2} \sum_{j=0}^{N-1} \hat{\Psi}_{j}^{\nu \dagger} \mathcal{H}_{\nu} \hat{\Psi}_{j}^{\nu}+\frac{1}{2} \sum_{j=0}^{N-2}\left[\hat{\Psi}_{j}^{\nu \dagger} T_{\nu} \hat{\Psi}_{j+1}^{\nu}+\hat{\Psi}_{j+1}^{\nu \dagger} T_{\nu}^{\dagger} \hat{\Psi}_{j}^{\nu}\right],
$$

with the four-component spinor in site $j$ defined as $\hat{\Psi}_{j}^{\nu}=$ $\left(c_{j \uparrow}^{\nu}, c_{j \downarrow}^{\nu}, c_{j \downarrow}^{\nu \dagger},-c_{j \uparrow}^{v^{\dagger}}\right)^{\mathrm{T}}$ in the Nambu representation and

$$
\begin{aligned}
\mathcal{H}_{v} & =(2 t-\mu) \tau_{z} \otimes \sigma_{0}+B_{x} \tau_{0} \otimes \sigma_{x}+\Delta \tau_{x} \otimes \sigma_{0}, \\
T_{v} & =-t \tau_{z} \otimes \sigma_{0}+i \alpha \tau_{z} \otimes \sigma_{z},
\end{aligned}
$$

where $t$ is the hopping matrix element, $\mu$ is the chemical potential, $\Delta$ is the superconducting order parameter, $\alpha$ is the spin-orbit coupling, and $B_{x}$ is the Zeeman field [31]. The Pauli matrices $\tau_{a}\left(\sigma_{a}\right)$ and the identity $\tau_{0}\left(\sigma_{0}\right)$ act in particlehole (spin) space. The junction's tunneling Hamiltonian $\hat{H}_{\mathcal{T}}$ couples the leads only through their end site. To simplify the notation, we redefine $\hat{\Psi}_{N-1}^{1} \equiv \hat{\Psi}^{1}, \hat{\Psi}_{0}^{2} \equiv \hat{\Psi}^{2}$, and $\hat{\Psi}_{N-1}^{3} \equiv \hat{\Psi}^{3}$ and then write

$$
\begin{aligned}
& \hat{H}_{\mathcal{T}}=\frac{1}{2} \sum_{\nu \leqslant v^{\prime}}\left[\hat{\Psi}^{\nu \dagger} \mathcal{T}_{\nu v^{\prime}} \hat{\Psi}^{\nu^{\prime}}+\hat{\Psi}^{\nu^{\prime} \dagger} \mathcal{T}_{\nu v^{\prime}}^{\dagger} \hat{\Psi}^{\nu}\right], \\
& \mathcal{T}_{\nu v^{\prime}}=\left(\Gamma_{\nu v^{\prime}} e^{i\left(\tau_{z} \otimes \sigma_{0}\right)\left[\frac{\varphi_{\nu}-\varphi_{\nu^{\prime}}}{2}-\epsilon_{v v^{\prime}} \frac{\Phi}{3}\right]}+\delta_{\nu v^{\prime}} U\right) \tau_{z} \otimes \sigma_{0},
\end{aligned}
$$

with $\mathcal{T}_{v v^{\prime}}^{\dagger}=\mathcal{T}_{v^{\prime} v}$ and $\epsilon_{v v^{\prime}}$ the totally antisymmetric tensor such that $\epsilon_{12}=\epsilon_{23}=\epsilon_{31}=1$. The dimensionless parameter $\Phi$ describes a magnetic flux threading the junction (in units of the flux quantum $\Phi_{0}=h / e$ ) and $U$ is an on-site surface energy [31].

\section{TOPOLOGICAL AVERAGED TRANSCONDUCTANCE}

We are interested in computing the current $\left\langle\mathcal{J}_{\nu}\right\rangle$ flowing in lead $v$ when driving adiabatically the phase of a different lead $v^{\prime}$ with a time dependence given by Josephson's relation
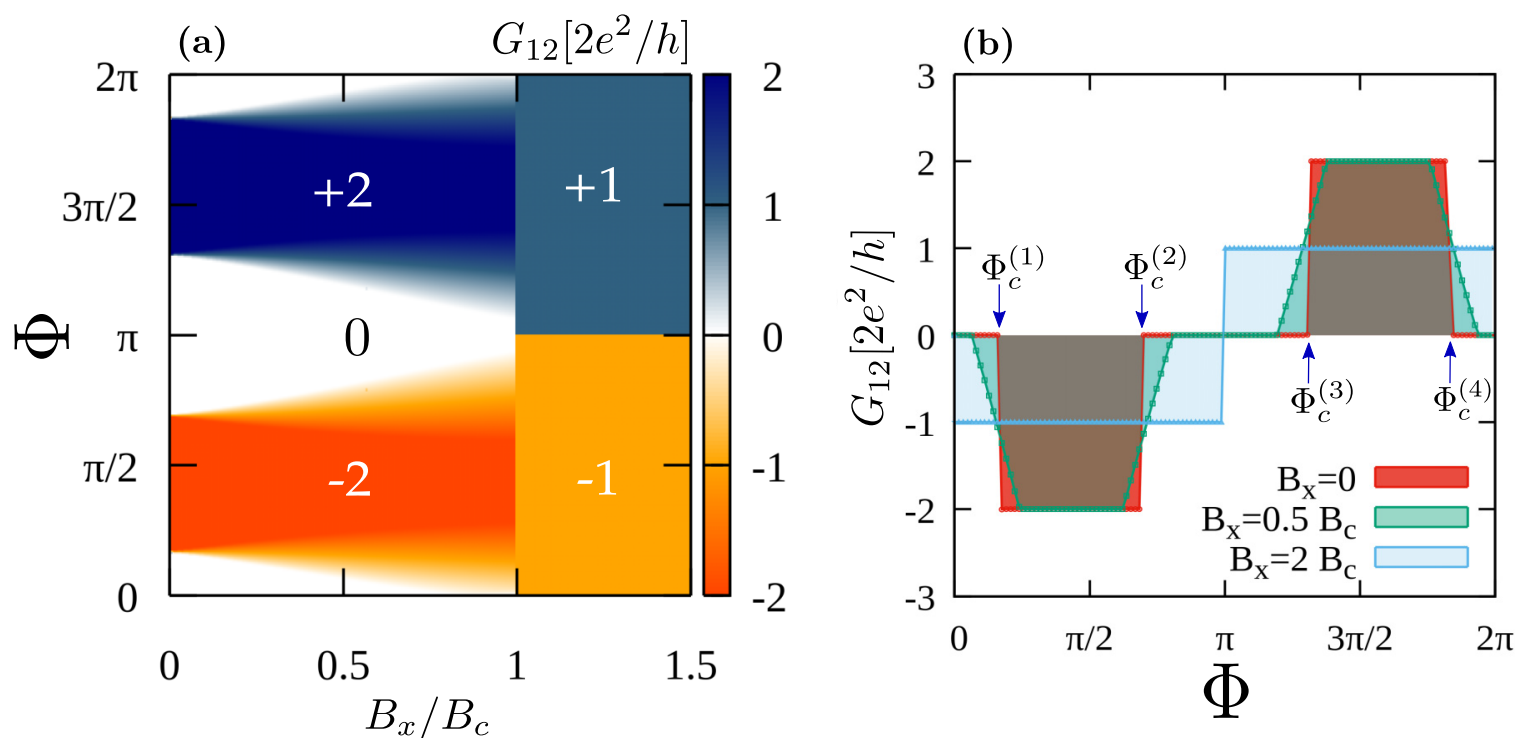

FIG. 2. (a) Zero-temperature average transconductance $G_{12}$ as a function of the external flux $\Phi$ and the in-plane Zeeman field $B_{x}$ (in units of the critical field $B_{c}=\sqrt{\Delta^{2}+\mu^{2}}$ ). (b) $G_{12}$ as a function of the external flux $\Phi$ for different values of $B_{x}$. Notice that the effective pumped charge $q^{\star}$ changes from $2 e$ to $e$ when $B_{x}$ becomes larger than $B_{c}$ ( $p$-wave or Majorana phase). 
$\dot{\varphi}_{v^{\prime}}=2 e V_{v^{\prime}} / \hbar$. The adiabatic correction to the current expectation value can be calculated using Wigner's representation of the two-time out-of-equilibrium Green's functions and performing a gradient expansion in the slow timescale (see Appendix A for details). The ATC results in

$$
G_{\nu v^{\prime}}=\frac{1}{4 \pi^{2}} \int_{0}^{2 \pi} d \varphi_{1} \int_{0}^{2 \pi} d \varphi_{2} \frac{\partial\left\langle\mathcal{J}_{\nu}\right\rangle}{\partial V_{\nu^{\prime}}}=-\frac{2 e^{2}}{h} W_{\nu v^{\prime}},
$$

where

$$
\begin{aligned}
W_{\nu v^{\prime}}= & \frac{1}{8 \pi^{2}} \int d^{2} \varphi \int d \omega \operatorname{Tr}\left[\mathcal{G}^{-1} \frac{\partial \mathcal{G}}{\partial \varphi_{\nu}} \mathcal{G}^{-1} \frac{\partial \mathcal{G}}{\partial \varphi_{\nu^{\prime}}}\right. \\
& \left.\times \mathcal{G}^{-1} \frac{\partial \mathcal{G}}{\partial \omega}-v \leftrightarrow v^{\prime}\right]
\end{aligned}
$$

is the winding number associated with the Green's function $\mathcal{G}$, which is topologically quantized whenever there is a gap at the Fermi energy. The trace is performed on both spin and particle-hole spaces. In the above expressions, $\mathcal{G}\left(\omega,\left\{\varphi_{\nu}\right\}\right)$ is the Feynman Green's function of the entire Hamiltonian in the total adiabatic limit-it follows the perturbation, $\left\{\varphi_{v}(t)\right\}$, instantaneously at time $t$. This expression can be manipulated so as to be written in terms of the projected junction's Green function involving only the end sites of the nanowires $\left(\mathcal{G}_{J}\right)$; see Appendix A. We compute $\mathcal{G}_{J}$ numerically by a simple decimation procedure of the Hamiltonian defined in Eq. (1) (see Appendix B). Equations (4) and (5), originally derived in Ref. [17], generalize the ones derived in Ref. [12] since they provide, on an equal footing, the contribution to the ATC of both the ABS and the continuum states above the superconducting gap. In a more recent article, a similar expression in terms of the scattering matrix was obtained [32]. There are two points worth mentioning: (i) If both spin projections are degenerate, then the trace over these variables would simply result in a factor 2 , which means a transconductance quantization in units of $4 e^{2} / h$, and (ii) the Green's function formalism implicitly assumes a thermodynamic average (taken here at zero temperature), which means that if there is a band crossing of the ABS at zero energy, the transconductance will not necessarily be quantized.

\section{A. Topological phase diagram}

Figure 2 summarizes the main result of this work: (a) a color map of the thermal transconductance as a function of the external flux $\Phi$ and the in-plane Zeeman field $B_{x}$; (b) representative cuts of this color map for fixed $B_{x}$. For $B_{x}=0$ both spin projections are degenerate and four zero-energy Weyl points develop as a function of the external flux at $\Phi_{c}^{(i)}$ for $i=1,2,3,4$, marked with arrows in Fig. 2(b), making the ATC jump at these singular points between quantized values of $\pm 4 e^{2} / h$ and zero. As $B_{x}$ increases, the quantization does not persist at all flux values, which can only be due to band overlaps developing in the spectrum at zero energy, as we shall show below. At this point, we would like to emphasize that in this entire region $\left(B_{x}<B_{c}\right)$ the independent contributions to the ATC of both the ABS and the continuum spectrum are nontrivial and that our method adds them up to give the correct value. In some cases, it is possible to separate them by resorting to the so-called topological
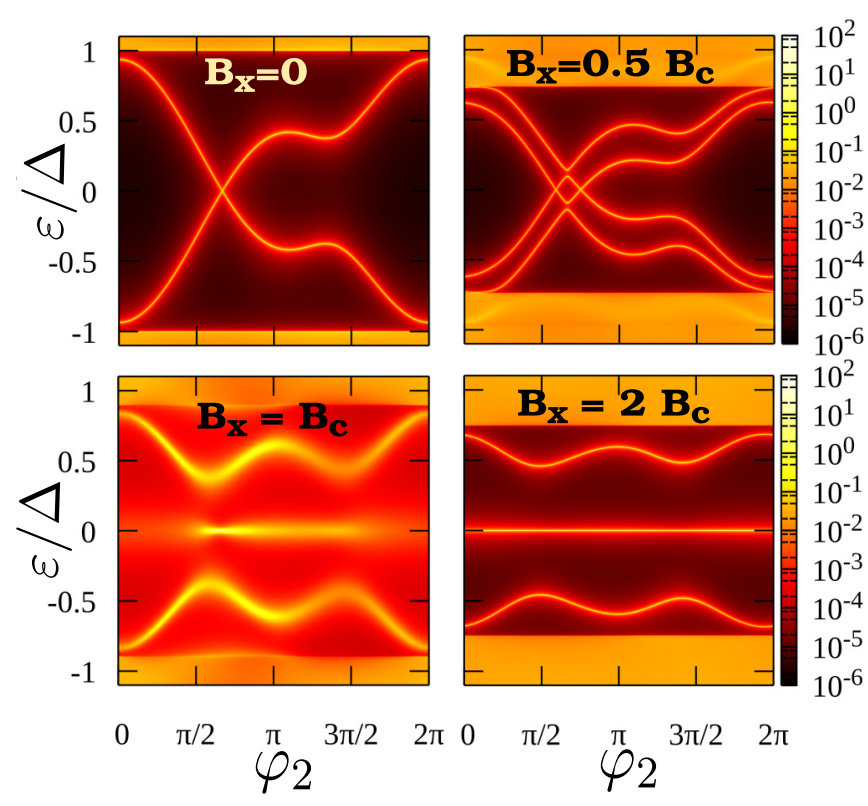

FIG. 3. Andreev subgap spectrum along the path $\varphi_{2}=-\varphi_{1}$ as a function of the Zeeman field $B_{x}$ for $\mu=3.2 \Delta$ and spin-orbit coupling $\alpha=50 \Delta$. The central magnetic flux was taken to be $\Phi_{c}^{(1)}=$ 0.53 .

Hamiltonian, defined as $H_{\text {top }}^{J}\left(\varphi_{1}, \varphi_{2}\right) \equiv-\mathcal{G}_{J}^{-1}(\omega=0)$, which has been proven $[33,34]$ to have the same topological properties as the system described by the entire $\mathcal{G}_{J}(\omega)$. Indeed, the Chern numbers calculated from the spectrum of $H_{\text {top }}^{J}$ do agree (see Appendix C) with the results presented in Fig. 2 and, for the particular case of $B_{x}=0$, with those obtained in Ref. [15].

The most striking result shown in Fig. 2 is manifested for $B_{x}>B_{c}$, where the ATC only takes quantized values in $2 e^{2} / h$. For these fields, Majorana degrees of freedom are expected to emerge in the system, with an effectively spinless superconductivity that causes the effective charge $q^{\star}$ to be that of the single electron.

\section{B. The junction's spectral density}

To understand these results, we show in Fig. 3 the spectral density of the trijunction $\mathcal{A}(\omega)=-1 / \pi \operatorname{Im}\left[\operatorname{Tr}\left(\mathcal{G}_{J}^{r}(\omega)\right)\right]$ along the path $\varphi_{2}=-\varphi_{1}$ for different values of $B_{x}$, where $\mathcal{G}_{J}^{r}(\omega)$ stands for the retarded junction Green's function. The external magnetic flux was taken to be $\Phi_{c}^{(1)}=0.53$, which corresponds to the first critical flux where a Weyl point appears in the ABS spectrum at zero magnetic field. As $B_{x}$ increases, the spin degeneracy is lifted and the ABSs overlap-as discussed in Ref. [35] — causing the ATC to be not quantized. At the critical field $B_{c}=\sqrt{\Delta^{2}+\mu^{2}}$ the superconducting gap closes, and for $B_{x}>B_{c}$ a zero-energy flat band emerges. The existence of this dispersionless state can be traced, as discussed below, to the fact that in the $p$-wave regime, Majorana modes $\hat{\gamma}_{v}$ develop at the end of each lead $v$. Only two effectively spinless dispersive ABSs coexist inside the gap with the Majorana band. Notice that in this three-terminal setup, no further requirement beyond thermal occupation is needed in order to observe the $2 e^{2} / h$ quantization since for any flux aside from 

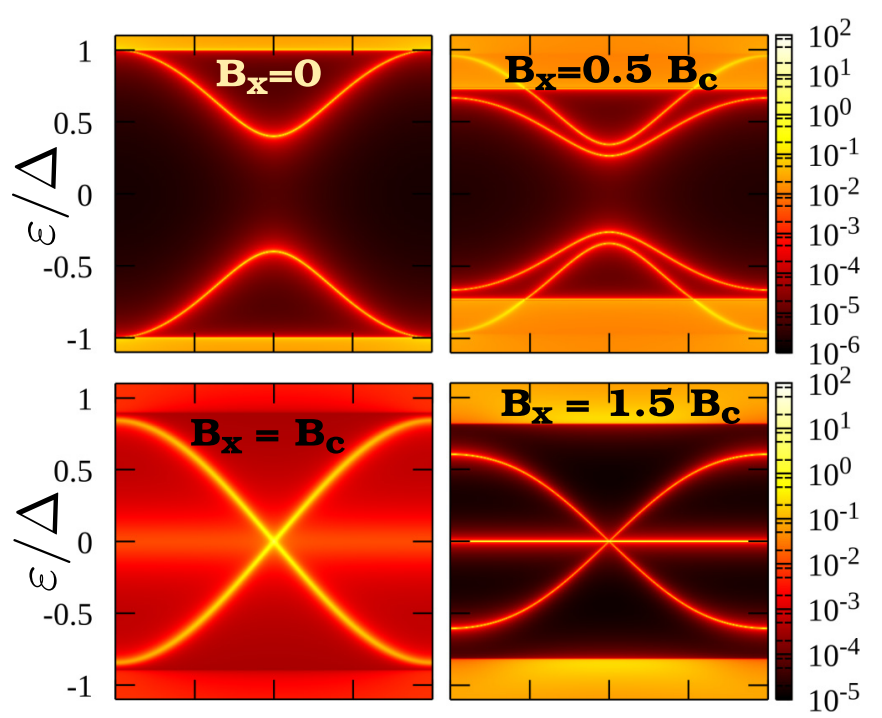

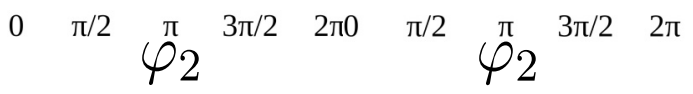

FIG. 4. Andreev subgap spectrum along $\varphi_{2}$ for $\varphi_{1}=2 \pi$ as a function of the Zeeman field $B_{x}$ for $\mu=3.2 \Delta$ and spin-orbit coupling $\alpha=50 \Delta$. The central magnetic flux was taken to be $\Phi=0$.

the critical ones the two ABSs never touch in the Majorana phase.

In Fig. 4 we show the spectral density along a different path $\left(\varphi_{1}=2 \pi\right)$ as a function of the Zeeman field for no external flux $(\Phi=0)$. Here, it becomes clear that the jump in the ATC from $-2 e^{2} / h$ to $2 e^{2} / h$ for $B_{x}>B_{c}$ can be traced back to the emergence of a pseudo-spin-1 Dirac-Weyl point, as is apparent in the figure for $B_{x}=1.5 B_{c}$. In this condition, when the external magnetic flux is turned on, it breaks the degeneracy between the two ABSs, making them detach from the zero-energy (Majorana) solution.

We notice that there are situations in which the ABSs are not detached from the continuum but they merge into it in the form of resonances (see Figs. 3 and 4) - this is so even in the Majorana regime (not shown). These mergings do not change the Green's function winding number since when two states become degenerate the Chern number of each one may change but their sum remains constant [36]. Hence, all changes in the quantized ATC presented in the topological phase diagram of Fig. 2 are due to the occurrence of crossings or Weyl points in the Andreev spectrum at zero energy.

In the $p$-wave phase $\left(B_{x}>B_{c}\right)$, the topological Hamiltonian $H_{\text {top }}$ is not well defined due to the presence of the Majorana bound state. However, for $B_{x} \gg B_{c}$, i.e., deep into the Kitaev regime, a simple low-energy model helps to describe and analyze the results. In this case, the tunneling Hamiltonian takes the form

$$
H_{T}^{\mathrm{eff}}\left(\varphi_{1}, \varphi_{2}\right)=i t_{12} \hat{\gamma}_{1} \hat{\gamma}_{2}+i t_{23} \hat{\gamma}_{2} \hat{\gamma}_{3}+i t_{31} \hat{\gamma}_{3} \hat{\gamma}_{1},
$$

with $\quad t_{12}=\tilde{\Gamma}_{12} \cos \left(\left(\varphi_{1}-\varphi_{2}\right) / 2-\Phi / 3\right), \quad t_{23}=$ $-\tilde{\Gamma}_{23} \cos \left(\varphi_{2} / 2-\Phi / 3\right), \quad t_{31}=-\tilde{\Gamma}_{13} \sin \left(\varphi_{1} / 2+\Phi / 3\right), \quad$ and $\tilde{\Gamma}_{i j}=\Gamma_{i j} / 2$. It possesses two particle-hole symmetric eigenvectors $\left|\psi_{ \pm}\right\rangle$with energies $E_{ \pm}= \pm \sqrt{t_{12}^{2}+t_{13}^{2}+t_{23}^{2}}$, which correspond to a finite-energy fermion (two Majorana degrees of freedom are paired up), and one zero-energy solution (the flat band) that is merely an "unpaired" Majorana mode living in the junction. Within this approximation, it is much easier to show that all the winding comes from the finite-energy fermionic band whose Chern number

$$
C_{-}=\frac{1}{8 \pi} \int_{0}^{4 \pi} d \varphi_{1} \int_{0}^{4 \pi} d \varphi_{2}\left[i\left\langle\partial_{\varphi_{1}} \psi_{-} \mid \partial_{\varphi_{2}} \psi_{-}\right\rangle-\left(\varphi_{1} \leftrightarrow \varphi_{2}\right)\right]
$$

changes from -1 to 1 at $\Phi=0$ and $\Phi=\pi$. In this regime, the continuum does not contribute. To further check these results, we have also made use of an analytic expression for the boundary Green's function for a Kitaev chain in the wide band limit [37] to calculate the winding number $W_{v v^{\prime}}$. Interestingly, we do recover the same results as in the spinful model, even though in this case the continuum has a smoother dependence in energy than in the complete model.

\section{CONCLUSIONS}

By expressing the average transconductance of a multiterminal JJ in terms of the Green's function of the junction, we have calculated the topological phase diagram of a threeterminal junction that completely describes the transition from the $s$-wave to the $p$-wave regime within a simple spinful model. In the former case, we found that transconductance is quantized in units of $4 e^{2} / h$, whenever the $\mathrm{ABS}$ spectrum is gapped, while in the latter it is in units of $2 e^{2} / h$. This is consistent with a change of the pumped charge over a Josephson period from $2 e$ to $e$, a distinctive signature of the fractional Josephson effect. Notably, in our odd-terminal setup this occurs under thermal occupation conditions-this is not the case in a four-terminal JJ; see Ref. [28].

As mentioned above, the Green's-function formalism used to calculate the winding number turns out to be essential to properly account for the contribution of both the Andreev subgap levels and the continuum states, which are, in general, unavoidably mixed. In addition, this method possesses several important advantages. On the one hand, it can in principle incorporate the effect of interactions, provided a good approximation for the single-particle Green's function is obtained. It can also properly handle the presence of an uncoupled Majorana state always pinned at zero energy. On the other hand, the method could easily incorporate microscopic details of the junction through more realistic calculations of the junction's Green's functions that might be relevant for a proper comparison with experimental data.

\section{ACKNOWLEDGMENTS}

We acknowledge financial support from ANPCyT (Grants No. PICTs 2013-1045 and No. 2016-0791), from CONICET (Grant No. PIP 11220150100506), and from SeCyT-UNCuyo (Grant No. 06/C526).

\section{APPENDIX A: ADIABATIC EXPANSION OF THE JOSEPHSON CURRENT}

We present here, for the sake of completeness, the expression derived in Ref. [17] to calculate the topological 
transconductance of a multiterminal Josephson junction. In general, the adiabatic expansion of the Josephson current can be done directly with the Green's function of the junction's Hamiltonian. We begin with Dyson's equation of motion of the Green's function of the entire (noninteracting) Hamiltonian $H(t)$ describing the junction and the leads written in the Bogoliubov-de Gennes (BdG) representation of particle-hole and spin spaces used in the main text,

$$
\left[i \partial_{t}-H(t)\right] \hat{\mathcal{G}}\left(t, t^{\prime}\right)=\delta\left(t-t^{\prime}\right),
$$

where the time dependence appears only on the tunneling Hamiltonian throughout the superconducting phases. The approach is based on the separation of slow and fast timescales in Dyson's equation by taking advantage of Wigner's representation. Under this formalism, two time variables, the relative time $t_{\text {rel }}=t-t^{\prime}$ and the average one $t_{\mathrm{av}}=\frac{t+t^{\prime}}{2}$, are handled. Time derivatives of the former are used as a small parameter, making feasible a perturbation scheme. Wigner's representation is given by

$$
\begin{aligned}
\hat{\mathcal{G}}\left(t, t^{\prime}\right) & =\int_{-\infty}^{\infty} \frac{d \omega}{2 \pi} e^{-i \omega\left(t-t^{\prime}\right)} \widetilde{\mathcal{G}}\left(\omega, t_{\mathrm{av}}\right), \\
\widetilde{\mathcal{G}}\left(\omega, t_{\mathrm{av}}\right) & =\int_{-\infty}^{\infty} d t_{\mathrm{rel}} e^{i \omega t_{\mathrm{rel}}} \hat{\mathcal{G}}\left(t, t^{\prime}\right) .
\end{aligned}
$$

Taking into account that $\partial_{t}=\frac{1}{2} \partial_{t_{\mathrm{av}}}+\partial_{t_{\mathrm{rel}}}$, Eq. (A1) can be written to all orders as [38]

$$
\left[\omega+\frac{i}{2} \partial_{t_{\mathrm{av}}}-e^{\frac{1}{2 i} \partial_{\omega}^{\mathcal{G}} \partial_{t_{\mathrm{av}}}^{H}} H\left(t_{\mathrm{av}}\right)\right] \widetilde{\mathcal{G}}\left(\omega, t_{\mathrm{av}}\right)=\mathcal{I}
$$

where we applied the following manipulation:

$$
\begin{aligned}
& \int_{-\infty}^{\infty} e^{i \omega t_{\mathrm{rel}}} H\left(t_{\mathrm{av}}+t_{\mathrm{rel}} / 2\right) \hat{\mathcal{G}}\left(t_{\mathrm{av}}+\frac{t_{\mathrm{rel}}}{2}, t_{\mathrm{av}}-\frac{t_{\mathrm{rel}}}{2}\right) d t_{\mathrm{rel}} \\
& =\int_{-\infty}^{\infty} e^{i \omega t_{\mathrm{rel}}} e^{\frac{t_{\mathrm{rel}}}{2} \partial_{t_{\mathrm{av}}}^{H}} H\left(t_{\mathrm{av}}\right) \hat{\mathcal{G}}\left(t_{\mathrm{av}}+\frac{t_{\mathrm{rel}}}{2}, t_{\mathrm{av}}-\frac{t_{\mathrm{rel}}}{2}\right) d t_{\mathrm{rel}} \\
& =\int_{-\infty}^{\infty} e^{\frac{1}{2 i} \partial_{\omega} \partial_{t_{\mathrm{av}}}^{H}} e^{i \omega t_{\mathrm{rel}}} H\left(t_{\mathrm{av}}\right) \hat{\mathcal{G}}\left(t_{\mathrm{av}}+\frac{t_{\mathrm{rel}}}{2}, t_{\mathrm{av}}-\frac{t_{\mathrm{rel}}}{2}\right) d t_{\mathrm{rel}} \\
& =e^{\frac{1}{2 i} \partial_{\omega}^{\mathcal{G}} \partial_{t_{\mathrm{av}}}^{H} H\left(t_{\mathrm{av}}\right) \widetilde{\mathcal{G}}\left(\omega, t_{\mathrm{av}}\right) .}
\end{aligned}
$$

We are interested in a first-order approximation, where the Green's function is perturbed up to first derivatives of the average time. In that case, Eq. (A3) is given by

$$
\left(\omega+\frac{i}{2} \partial_{t_{\mathrm{av}}}-H\left(t_{\mathrm{av}}\right)-\frac{1}{2 i} \frac{\partial H\left(t_{\mathrm{av}}\right)}{\partial t_{\mathrm{av}}} \partial_{\omega}\right) \widetilde{\mathcal{G}}\left(\omega, t_{\mathrm{av}}\right)=\mathcal{I} \text {. }
$$

Introducing a series expansion for the Green's function $\widetilde{\mathcal{G}}\left(\omega, t_{\mathrm{av}}\right)=\sum_{n} \widetilde{\mathcal{G}}^{(n)}$, we can obtain the next system of equations,

$$
\begin{gathered}
(\omega-H) \widetilde{\mathcal{G}}^{(0)}=\mathcal{I}, \\
(\omega-H) \widetilde{\mathcal{G}}^{(1)}=\frac{1}{2 i} \partial_{t_{\mathrm{av}}} H \partial_{\omega} \widetilde{\mathcal{G}}^{(0)}+\frac{1}{2 i} \partial_{t_{\mathrm{av}}} \widetilde{\mathcal{G}}^{(0)},
\end{gathered}
$$

where the arguments $\left(\omega, t_{\mathrm{av}}\right)$ are omitted for the sake of brevity. Solving Eq. (A6) and substituting it into Eq. (A7), we get

$$
\begin{gathered}
\widetilde{\mathcal{G}}^{(0)}=(\omega-H)^{-1}, \\
\widetilde{\mathcal{G}}^{(1)}=\frac{1}{2 i} \widetilde{\mathcal{G}}^{(0)}\left[\widetilde{\mathcal{G}}^{(0)}, \partial_{t_{\mathrm{av}}} H \widetilde{\mathcal{G}}^{(0)}\right],
\end{gathered}
$$

where $[\cdot, \cdot]$ is the commutator. We have also used the properties of the derivatives of inverse functions $\partial_{\lambda} A^{-1}=$ $-A^{-1} \partial_{\lambda} A A^{-1}$, meaning in this case that

$$
\begin{gathered}
\partial_{\omega} \widetilde{\mathcal{G}}^{(0)}=-\widetilde{\mathcal{G}}^{(0)} \widetilde{\mathcal{G}}^{(0)}, \\
\partial_{t_{\mathrm{av}}} \widetilde{\mathcal{G}}^{(0)}=\widetilde{\mathcal{G}}^{(0)} \partial_{t_{\mathrm{av}}} H \widetilde{\mathcal{G}}^{(0)} .
\end{gathered}
$$

The mean value of a single-particle operator written in the BdG basis $\hat{O}(t)=\frac{1}{2} \sum_{\alpha \beta} \hat{\Psi}_{\beta}^{\dagger}(t) O_{\beta \alpha} \hat{\Psi}_{\alpha}(t)$ is given by

$$
\begin{aligned}
\left\langle\phi_{0}|\hat{O}(t)| \phi_{0}\right\rangle & =\frac{1}{2} \lim _{t^{\prime}-t \rightarrow \epsilon^{+}} \sum_{\alpha \beta} O_{\beta \alpha}\left\langle\phi_{0}\left|\hat{\Psi}_{\beta}^{\dagger}\left(t^{\prime}\right) \hat{\Psi}_{\alpha}(t)\right| \phi_{0}\right\rangle \\
& =-\frac{i}{2} \lim _{t^{\prime}-t \rightarrow \epsilon^{+}} \sum_{\alpha \beta} O_{\beta \alpha} \mathcal{G}_{\alpha \beta}\left(t, t^{\prime}\right) \\
& =-\frac{i}{2} \lim _{t^{\prime}-t \rightarrow \epsilon^{+}} \operatorname{Tr}\left[O \hat{\mathcal{G}}\left(t, t^{\prime}\right)\right]
\end{aligned}
$$

By writing Eq. (A12) in Wigner's representation and expanding the Green's function up to first order, we obtain

$$
\langle O(t)\rangle \simeq \frac{1}{2} \lim _{t^{\prime}-t \rightarrow \epsilon^{+}}\left\{-i \int_{-\infty}^{\infty} \frac{d \omega}{2 \pi} \operatorname{Tr}\left[O \widetilde{\mathcal{G}}^{(0)}\right]+\int_{-\infty}^{\infty} \frac{d \omega}{4 \pi} \operatorname{Tr}\left(\frac{\partial H}{\partial t_{\mathrm{av}}} \widetilde{\mathcal{G}}^{(0)} O \frac{\partial \widetilde{\mathcal{G}}^{(0)}}{\partial \omega}-O \widetilde{\mathcal{G}}^{(0)} \frac{\partial H}{\partial t_{\mathrm{av}}} \frac{\partial \widetilde{\mathcal{G}}^{(0)}}{\partial \omega}\right)\right\} .
$$

By specifying $O$ as the current operator $\mathcal{J}_{v}=2 e \partial_{\varphi_{v}} H$ and using that $\lim _{t^{\prime}-t \rightarrow \epsilon^{+}} \partial_{t_{\mathrm{av}}} H=\sum_{v^{\prime}} \partial_{\varphi_{v^{\prime}}} H \dot{\varphi}_{\nu^{\prime}}(t)$, we get the first-order correction to the current expectation value given by

$$
\begin{aligned}
\left\langle\mathcal{J}_{\nu}^{(1)}(t)\right\rangle & =e \sum_{\nu^{\prime}} \int_{-\infty}^{\infty} \frac{d \omega}{4 \pi} \operatorname{Tr}\left[\partial_{\varphi_{v^{\prime}}} H \cdot \widetilde{\mathcal{G}}^{(0)} \partial_{\varphi_{\nu}} H \cdot \partial_{\omega} \widetilde{\mathcal{G}}^{(0)}-v^{\prime} \leftrightarrow v\right] \dot{\varphi}_{v^{\prime}}(t) \\
& =-\frac{2 e^{2}}{\hbar} \sum_{\nu^{\prime}} \int_{-\infty}^{\infty} \frac{d \omega}{4 \pi} \operatorname{Tr}\left[\widetilde{\mathcal{G}}^{(0)-1} \partial_{\varphi_{\nu}} \widetilde{\mathcal{G}}^{(0)} \cdot \widetilde{\mathcal{G}}^{(0)-1} \partial_{\varphi_{\nu^{\prime}}} \mathcal{G}^{(0)} \cdot \widetilde{\mathcal{G}}^{(0)-1} \partial_{\omega} \widetilde{\mathcal{G}}^{(0)}-v \leftrightarrow v^{\prime}\right] V_{\nu^{\prime}}
\end{aligned}
$$


The averaged transconductance then simply results in

$$
G_{\nu \nu^{\prime}}=\frac{1}{4 \pi^{2}} \int_{0}^{2 \pi} d \varphi_{1} \int_{0}^{2 \pi} d \varphi_{2} \frac{\partial\left\langle\mathcal{J}_{\nu}^{(1)}\right\rangle}{\partial V_{\nu^{\prime}}},
$$

which leads to Eq. (4) used to obtain our main results. We then recover an expression of this adiabatic transport coefficient in terms of the Green's-function winding number. By noticing that $\widetilde{\mathcal{G}}^{(0)}\left(\omega, t_{\mathrm{av}}\right)$ depends on the average time $t_{\mathrm{av}}$ only throughout the superconducting phases, we can define $\mathcal{G}\left(\omega,\left\{\varphi_{\nu}\right\}\right)=\widetilde{\mathcal{G}}^{(0)}\left(\omega,\left\{\varphi_{\nu}\left(t_{\mathrm{av}}\right)\right\}\right)$. Furthermore, since the tunneling Hamiltonian acts locally on the end sites of each lead, this expression can be simplified so as to be written only in terms of the projected junction Green's function $\mathcal{G}_{J}$. The averaged transconductance can then be written as

$$
\begin{aligned}
G_{\nu v^{\prime}}= & -\frac{2 e^{2}}{h} \frac{1}{8 \pi^{2}} \int_{0}^{2 \pi} d^{2} \varphi \int d \omega \\
& \times \operatorname{Tr}_{J}\left[\partial_{\varphi_{\nu^{\prime}}} \mathcal{T} \cdot \mathcal{G}_{J} \cdot \partial_{\varphi_{v}} \mathcal{T} \cdot \partial_{\omega} \mathcal{G}_{J}-v^{\prime} \leftrightarrow v\right]
\end{aligned}
$$

where the phase derivatives have been explicitly written in terms of the tunneling Hamiltonian $\mathcal{T}$ as defined in Eq. (3), and the trace $\operatorname{Tr}_{J}$ acts locally on the junction's states. We emphasize that the result does not depend explicitly on the slow timescale anymore, since the instantaneous superconducting phases are integrated out.

\section{APPENDIX B: BOUNDARY GREEN'S FUNCTIONS OF THE UNCOUPLED LEADS BY DECIMATION}

The spinful boundary Green's function of each one of the leads is obtained by a numerical decimation procedure applied to the Hamiltonian

$\hat{H}=\frac{1}{2} \sum_{j=0}^{N-1} \hat{\Psi}_{j}^{\dagger} \mathcal{H} \hat{\Psi}_{j}+\frac{1}{2} \sum_{j=0}^{N-2}\left[\hat{\Psi}_{j}^{\dagger} T \hat{\Psi}_{j+1}+\hat{\Psi}_{j+1}^{\dagger} T^{\dagger} \hat{\Psi}_{j}\right]$,

where the four-component spinor in site $j$ is defined as $\hat{\Psi}_{j}=$ $\left(c_{j \uparrow}, c_{j \downarrow}, c_{j \downarrow}^{\dagger},-c_{j \uparrow}^{\dagger}\right)^{\mathrm{T}}$ in the Nambu representation and

$$
\begin{aligned}
\mathcal{H} & =(2 t-\mu) \tau_{z} \otimes \sigma_{0}+B_{x} \tau_{0} \otimes \sigma_{x}+\Delta \tau_{x} \otimes \sigma_{0}, \\
T & =-t \tau_{z} \otimes \sigma_{0}+i \alpha \tau_{z} \otimes \sigma_{z},
\end{aligned}
$$

where $t$ is the hopping matrix element, $\alpha$ is the spin orbitcoupling, and $B_{x}$ is the Zeeman field. The Pauli matrices $\tau_{a}\left(\sigma_{a}\right)$ and the identity $\tau_{0}\left(\sigma_{0}\right)$ act in particle-hole (spin) space. The decimation procedure iteratively eliminates at each step of the Green's functions equations of motion of even sites of the chain, eventually encapsulating numerous sites through a recursion relation using renormalized parameters. Assuming that the initial undressed Green's functions of the left boundary $g_{0}^{L}(\omega)$, the bulk $g_{0}^{B}(\omega)$, and the right boundary $g_{0}^{R}(\omega)$ are all the same and equal to $(\omega-\mathcal{H})^{-1}$, at step $n \geqslant 1$ of the decimation they take the form

$$
\begin{gathered}
g_{n}^{L}(\omega)=\left[\left[g_{n-1}^{L}(\omega)\right]^{-1}-T_{n-1} g_{n-1}^{B}(\omega) T_{n-1}^{\dagger}\right]^{-1}, \\
g_{n}^{B}(\omega)=\left[\left[g_{n-1}^{B}(\omega)\right]^{-1}-\Sigma_{n-1}(\omega)\right]^{-1}, \\
g_{n}^{R}(\omega)=\left[\left[g_{n-1}^{R}(\omega)\right]^{-1}-T_{n-1}^{\dagger} g_{n-1}^{B}(\omega) T_{n-1}\right]^{-1},
\end{gathered}
$$

where the renormalized hoppings are given by

$$
\begin{aligned}
T_{n} & =T_{n-1} g_{n-1}^{B}(\omega) T_{n-1}, \\
T_{n}^{\dagger} & =T_{n-1}^{\dagger} g_{n-1}^{B}(\omega) T_{n-1}^{\dagger},
\end{aligned}
$$
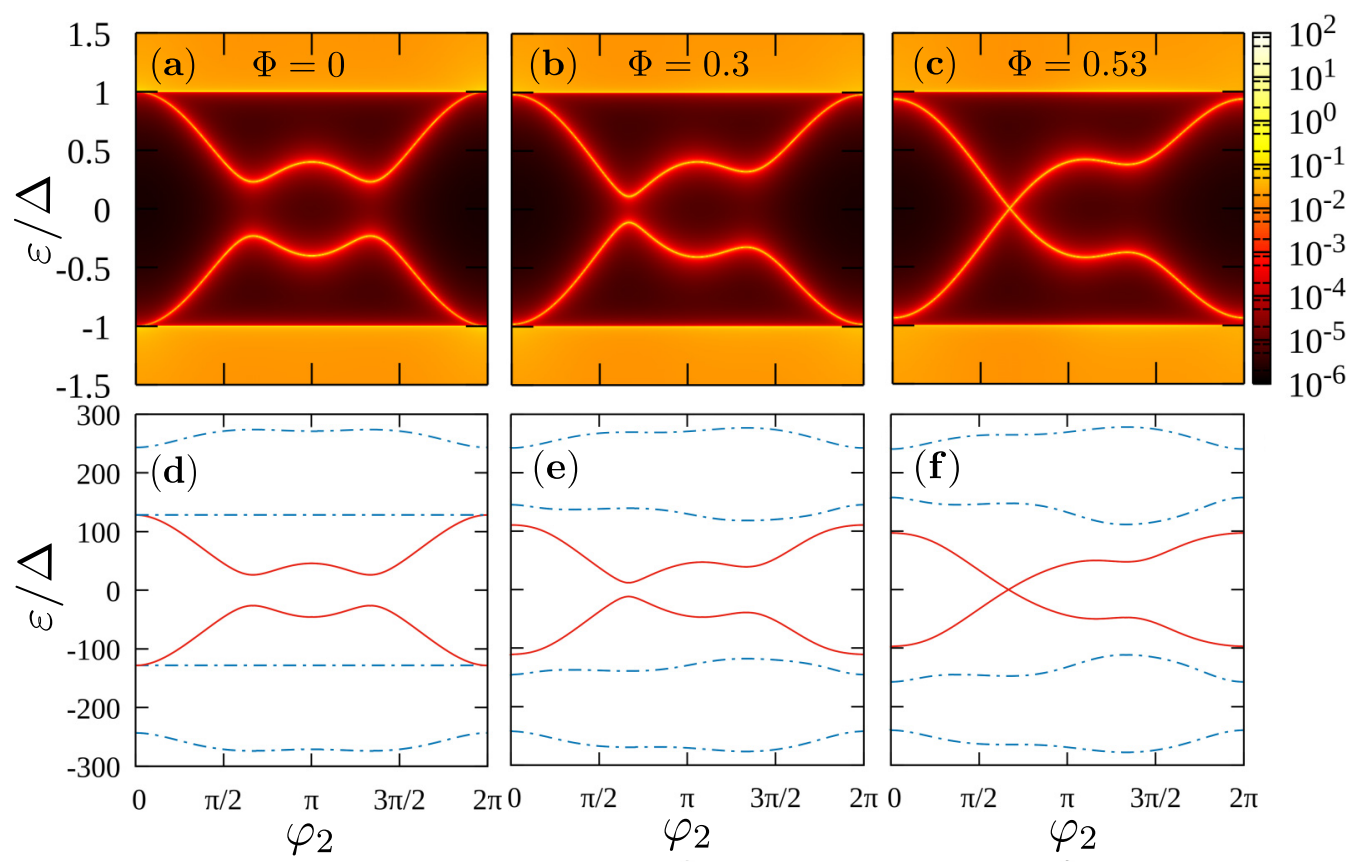

FIG. 5. Upper panels show the spectral density of the three-terminal Josephson junction in the spin-degenerate case $\left(B_{x}=0\right)$ along the path $\varphi_{2}=-\varphi_{1}$ for different fluxes threading the central scattering region between the leads (a) $\Phi=0$, (b) $\Phi=0.3$, and (c) $\Phi=0.53$. Lower panels (d), (e), and (f) show the eigenvalues of $H_{\text {top }}^{J}(\varphi)=-\mathcal{G}_{J}^{-1}(\omega=0)$ for this same path with the same corresponding fluxes. Solid lines indicate the bands topologically connected with the Andreev bound states, and dashed lines indicate the eigenvalues bearing the topological information of the continuum states. 
and $\quad \Sigma_{n-1}(\omega)=T_{n-1} g_{n-1}^{B}(\omega) T_{n-1}^{\dagger}+T_{n-1}^{\dagger} g_{n-1}^{B}(\omega) T_{n-1}$. The initial tunneling is just the undressed one $T_{n=0}=T$ [see Eq. (B2)]. For sufficiently large $n$, each of these Green's functions accurately represents the left and right boundary of a semi-infinite chain (generally not equal in topological systems) and the bulk of an infinite one.

These boundary Green's functions are used to construct the three-terminal junction Green's function (see Fig. 1) as $\mathcal{G}_{J}^{r / a}(\omega)=\left[g^{-1}(\omega \pm i \eta)-\mathcal{T}\right]^{-1}$, where the superscripts $r / a$ stand for retarded and advanced, respectively, $g(\omega)$ is a $12 \times$ 12 block-diagonal matrix of the form

$$
g(\omega)=\left[\begin{array}{ccc}
g^{R}(\omega) & \mathbf{0} & \mathbf{0} \\
\mathbf{0} & g^{L}(\omega) & \mathbf{0} \\
\mathbf{0} & \mathbf{0} & g^{R}(\omega)
\end{array}\right],
$$

and $\mathcal{T}$ is the tunneling between the leads as described in Eq. (3) in the main text. The Feynman Green's function used to calculate the winding number is then obtained as

$$
\mathcal{G}_{J}(\omega)=\mathcal{G}_{J}^{r}(\omega)+f(\omega)\left(\mathcal{G}_{J}^{a}(\omega)-\mathcal{G}_{J}^{r}(\omega)\right),
$$

with $f(\omega)$ the Fermi-Dirac distribution function.

\section{APPENDIX C: TOPOLOGICAL HAMILTONIAN FOR $B<B_{c}$ : CONTRIBUTIONS FROM THE ABS AND THE CONTINUUM STATES}

It was recently stated that, under quite general assumptions, all the topological information of the total $\mathcal{G}(\omega)$ of a system is encoded in the the so-called "topological Hamiltonian" defined as $H_{\text {top }} \equiv-\mathcal{G}^{-1}(\omega=0)[33,34]$. Notice this is a welldefined Hermitian operator, since the self-energy $\Sigma(\omega=0)$ has no imaginary part. The key idea is that the entire $\mathcal{G}(\omega)$ can be smoothly deformed to $\mathcal{G}_{\text {eff }}(\omega)=\left[\omega+\mathcal{G}^{-1}(\omega=0)\right]^{-1}$ preserving its topology. In this case, it is easy to prove that the winding number of the Green's function in a given parameter space $\left(\varphi_{1}, \varphi_{2}\right)$ can be calculated with the Chern numbers associated with each of the eigenstates $\left|\psi_{n}\right\rangle$ of $H_{\text {top }}$ as

$$
W_{12}=\sum_{n \in \mathrm{occ}} \mathcal{C}_{12}^{n}
$$

where the sum runs over all occupied states and

$\left.C_{12}^{n}=\frac{1}{2 \pi} \int_{0}^{2 \pi} d \varphi_{1} \int_{0}^{2 \pi} d \varphi_{2}\left[i\left|\partial_{\varphi_{1}} \psi_{n}\right| \partial_{\varphi_{2}} \psi_{n}\right\rangle-\left(\varphi_{1} \leftrightarrow \varphi_{2}\right)\right]$.

This theoretical tool provides a much simpler way of obtaining the topological invariants without the need of an integral over frequency space [see Eq. (5) in the main text].

In the case of Josephson junctions, the eigenstates resulting from diagonalizing $H_{\text {top }}^{J} \equiv-\mathcal{G}_{J}^{-1}(\omega=0)$ should then reflect the topological nature of both the Andreev subgap structure and the continuum states above the superconducting gap, as we shall show below. There is only one caveat: the method is only applicable in our system for in-plane magnetic fields below the critical value $B_{x}<B_{c}$, since for larger fields-after the topological transition-an unpaired Majorana emerges at zero energy (the "flat band"). In this case, the Green's function is singular at $\omega=0$ and hence not invertible.

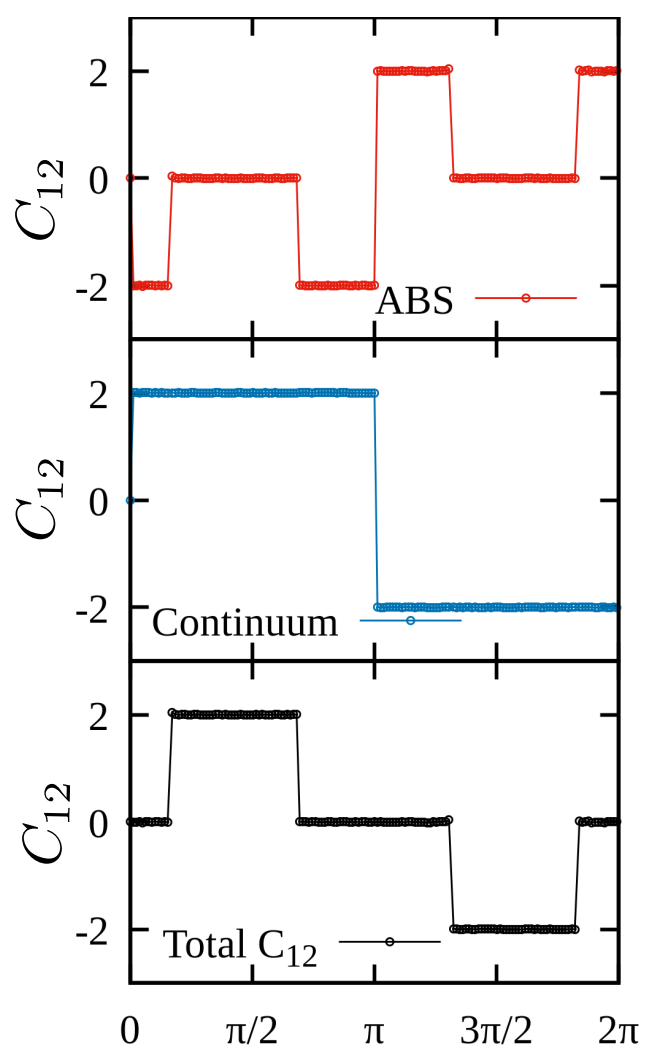

FIG. 6. Chern numbers $C_{12}$ of the Andreev bound states (ABS) and the continuum states. The lower panel shows the sum of both contributions.

In the upper panels of Fig. 5 we present the spectral density of the trijunction $\mathcal{A}(\omega)=-1 / \pi \operatorname{Im}\left[\operatorname{Tr}\left(\mathcal{G}_{J}^{r}(\omega)\right)\right]$ along the path $\varphi_{2}=-\varphi_{1}$ for different values of the fluxes threading the central scattering region between the superconducting leads. The in-plane magnetic field was chosen to be zero $\left(B_{x}=0\right)$ so that the eigenenergies are spin-degenerate. The lower panels show the outcome of diagonalizing the topological Hamiltonian for the corresponding fluxes. Six particle-hole symmetric and spin-degenerate bands are present. The eigenvalues in solid lines, which are closer to the Fermi level, should possess the topological information of the Andreev subgap states, while the dashed eigenvalues should represent the continuum states. Note that even though the energy scale is very different from the original one, the touching of the lowest ABS with the continuum at $\varphi_{1}=\varphi_{2}=0$ and zero external flux $\Phi=0$ [see Figs. 5(a) and 5(d)] is accurately described, as well as the closing of the gap between the ABS at the first critical flux $\Phi=\Phi_{c}^{(1)}=0.53$ [see Figs. 5(c) and 5(f)].

In Fig. 6 we show the Chern numbers of these bands as a function of the external flux $\Phi$. The Chern associated to the ABS (upper panel in Fig. 6) is obtained from the band closer to the Fermi level, while the one related to the continuum (middle panel in Fig. 6) is just the sum of the Cherns of the two lower bands in Fig. 5. All of them are spin-degenerate, and, as a result, they lead to a simple factor 2 in their respective topological invariants. The sum of this contributions (lower panel in Fig. 6) is precisely the winding number of the full frequency-dependent Green's function obtained in the main 
text, revealing the validity of the method. Notice also that the contributions from the ABS agree well with the calculation of Ref. [15].

This same procedure can be done for every $0<B_{x}<B_{c}$, where the spin degeneracy is lifted. The topological Hamiltonian $H_{\text {top }}^{J}$ possesses in this case 12 different particle-hole symmetric eigenvalues. In this regime, the contribution from the continuum states and the Andreev levels cannot be separated, since the gap closing makes one of the ABSs merge with the continuum. Nonetheless, the sum of all contributions up to the Fermi level correctly recovers the $s$-wave part of the phase diagram presented in Fig. 2(a) of the main text.
[1] J. Alicea, New directions in the pursuit of Majorana fermions in solid state systems, Rep. Prog. Phys. 75, 076501 (2012).

[2] R. Aguado, Majorana quasiparticles in condensed matter, Riv. Nuovo Cimento 40, 523 (2017).

[3] M. Sato and Y. Ando, Topological superconductors: A review, Rep. Prog. Phys. 80, 076501 (2017).

[4] T. Ozawa, H. M. Price, A. Amo, N. Goldman, M. Hafezi, L. Lu, M. C. Rechtsman, D. Schuster, J. Simon, O. Zilberberg, and I. Carusotto, Topological photonics, Rev. Mod. Phys. 91, 015006 (2019).

[5] T. Oka and H. Aoki, Photovoltaic Hall effect in graphene, Phys. Rev. B 79, 081406(R) (2009).

[6] T. Kitagawa, E. Berg, M. Rudner, and E. Demler, Topological characterization of periodically driven quantum systems, Phys. Rev. B 82, 235114 (2010).

[7] N. H. Lindner, G. Refael, and V. Galitski, Floquet topological insulator in semiconductor quantum wells, Nat. Phys. 7, 490 (2011).

[8] I. Bloch, J. Dalibard, and S. Nascimbène, Quantum simulations with ultracold quantum gases, Nat. Phys. 8, 267 (2012).

[9] G. Jotzu, M. Messer, R. Desbuquois, M. Lebrat, T. Uehlinger, D. Greif, and T. Esslinger, Experimental realization of the topological Haldane model with ultracold fermions, Nature (London) 515, 237 (2014).

[10] M. Aidelsburger, M. Lohse, C. Schweizer, M. Atala, J. T. Barreiro, S. Nascimbène, N. R. Cooper, I. Bloch, and N. Goldman, Measuring the Chern number of Hofstadter bands with ultracold bosonic atoms, Nat. Phys. 11, 162 (2014).

[11] N. R. Cooper, J. Dalibard, and I. B. Spielman, Topological bands for ultracold atoms, Rev. Mod. Phys. 91, 015005 (2019).

[12] R.-P. Riwar, M. Houzet, J. S. Meyer, and Y. V. Nazarov, Multi-terminal Josephson junctions as topological matter, Nat. Commun. 7, 11167 (2016).

[13] J. E. Avron, A. Raveh, and B. Zur, Adiabatic quantum transport in multiply connected systems, Rev. Mod. Phys. 60, 873 (1988).

[14] J. E. Avron and M. C. Cross, Integer charge transport in Josephson junctions, Phys. Rev. B 39, 756 (1989).

[15] J. S. Meyer and M. Houzet, Nontrivial Chern Numbers in Three-Terminal Josephson Junctions, Phys. Rev. Lett. 119, 136807 (2017).

[16] H.-Y. Xie, M. G. Vavilov, and A. Levchenko, Topological Andreev bands in three-terminal Josephson junctions, Phys. Rev. B 96, 161406(R) (2017).

[17] L. Peralta Gavensky, G. Usaj, D. Feinberg, and C. A. Balseiro, Berry curvature tomography and realization of topological Haldane model in driven three-terminal Josephson junctions, Phys. Rev. B 97, 220505(R) (2018).

[18] R. L. Klees, G. Rastelli, J. C. Cuevas, and W. Belzig, Microwave spectroscopy reveals the quantum geometric tensor of topological Josephson matter, arXiv:1810.11277.
[19] E. Strambini, S. D’Ambrosio, F. Vischi, F. S. Bergeret, Y. V. Nazarov, and F. Giazotto, The $\omega$-SQUIPT as a tool to phaseengineer Josephson topological materials, Nat. Nanotechnol. 11, 1055 (2016).

[20] F. Vischi, M. Carrega, E. Strambini, S. D’Ambrosio, F. S. Bergeret, Y. V. Nazarov, and F. Giazotto, Coherent transport properties of a three-terminal hybrid superconducting interferometer, Phys. Rev. B 95, 054504 (2017).

[21] A. W. Draelos, M.-T. Wei, A. Seredinski, H. Li, Y. Mehta, K. Watanabe, T. Taniguchi, I. V. Borzenets, F. Amet, and G. Finkelstein, Supercurrent flow in multiterminal graphene Josephson junctions, Nano Lett. 19, 1039 (2019).

[22] N. Pankratova, H. Lee, R. Kuzmin, M. Vavilov, K. Wickramasinghe, W. Mayer, J. Yuan, J. Shabani, and V. E. Manucharyan, The multi-terminal Josephson effect, arXiv: 1812.06017.

[23] E. Eriksson, R.-P. Riwar, M. Houzet, J. S. Meyer, and Y. V. Nazarov, Topological transconductance quantization in a four-terminal Josephson junction, Phys. Rev. B 95, 075417 (2017).

[24] Y. Oreg, G. Refael, and F. von Oppen, Helical Liquids and Majorana Bound States in Quantum Wires, Phys. Rev. Lett. 105, 177002 (2010).

[25] R. M. Lutchyn, J. D. Sau, and S. Das Sarma, Majorana Fermions and a Topological Phase Transition in Semiconductor-Superconductor Heterostructures, Phys. Rev. Lett. 105, 077001 (2010).

[26] J. Wiedenmann, E. Bocquillon, R. S. Deacon, S. Hartinger, O. Herrmann, T. M. Klapwijk, L. Maier, C. Ames, C. Brüne, C. Gould, A. Oiwa, K. Ishibashi, S. Tarucha, H. Buhmann, and L. W. Molenkamp, $4 \pi$-periodic Josephson supercurrent in HgTe-based topological Josephson junctions, Nat. Commun. 7, 10303 (2016).

[27] D. Laroche, D. Bouman, D. J. van Woerkom, A. Proutski, C. Murthy, D. I. Pikulin, C. Nayak, R. J. J. van Gulik, J. Nygård, P. Krogstrup, L. P. Kouwenhoven, and A. Geresdi, Observation of the $4 \pi$-periodic Josephson effect in indium arsenide nanowires, Nat. Commun. 10, 245 (2019).

[28] M. Houzet and J. S. Meyer, Majorana-Weyl crossings in topological multi-terminal junctions, arXiv:1810.09962.

[29] H.-Y. Xie and A. Levchenko, Topological supercurrents interaction and fluctuations in the multiterminal Josephson effect, Phys. Rev. B 99, 094519 (2019).

[30] O. Deb, K. Sengupta, and D. Sen, Josephson junctions of multiple superconducting wires, Phys. Rev. B 97, 174518 (2018).

[31] In terms of the original continuous model, $t=\frac{\hbar^{2}}{2 m^{*} d^{2}}, \alpha=$ $\frac{\hbar \alpha_{\mathrm{so}}}{d}$, and $B_{x}=\frac{g \mu_{B} b_{x}}{2}$. To select reasonable parameters, we first choose the lattice spacing to be $d=2 \mathrm{~nm}$ and the effective mass $m^{*} \simeq 0.02 m_{e}$ for InAs, which yields the nearest-neighbor hopping $t=0.47 \mathrm{eV}$. We estimate $\alpha \simeq 10 \mathrm{meV}$, consistent with 
the measurements of Refs. [39,40] of a spin-orbit interaction length $l_{\text {so }} \approx 100 \mathrm{~nm}$. In the calculations, we refer the energy scale to the superconducting gap $\Delta=0.2 \mathrm{meV}$. The rest of the parameters are $\Gamma_{v v^{\prime}}=100 \Delta, U=-t$, and $\mu=3.2 \Delta$.

[32] E. V. Repin, Y. Chen, and Y. V. Nazarov, Topological properties of multiterminal superconducting nanostructures: Effect of a continuous spectrum, Phys. Rev. B 99, 165414 (2019).

[33] Z. Wang and S.-C. Zhang, Simplified Topological Invariants for Interacting Insulators, Phys. Rev. X 2, 031008 (2012).

[34] Z. Wang and B. Yan, Topological Hamiltonian as an exact tool for topological invariants, J. Phys.: Condens. Matter 25, 155601 (2013).

[35] T. Yokoyama and Y. V. Nazarov, Singularities in the Andreev spectrum of a multiterminal Josephson junction, Phys. Rev. B 92, 155437 (2015).
[36] J. E. Avron, R. Seiler, and B. Simon, Homotopy and Quantization in Condensed Matter Physics, Phys. Rev. Lett. 51, 51 (1983).

[37] A. Zazunov, R. Egger, and A. Levy Yeyati, Low-energy theory of transport in Majorana wire junctions, Phys. Rev. B 94, 014502 (2016).

[38] V. F. Kershaw and D. S. Kosov, Nonequilibrium Green's function theory for nonadiabatic effects in quantum electron transport, J. Chem. Phys. 147, 224109 (2017).

[39] P. Roulleau, T. Choi, S. Riedi, T. Heinzel, I. Shorubalko, T. Ihn, and K. Ensslin, Suppression of weak antilocalization in InAs nanowires, Phys. Rev. B 81, 155449 (2010).

[40] S. E. Hernández, M. Akabori, K. Sladek, C. Volk, S. Alagha, H. Hardtdegen, M. G. Pala, N. Demarina, D. Grützmacher, and T. Schäpers, Spin-orbit coupling and phase coherence in InAs nanowires, Phys. Rev. B 82, 235303 (2010). 